\title{
Evaluation of Acoustic Forces on A Particle in Aerosol Medium
}

Richard A. Dimenna and Si Young Lee

September 2007

Washington Savannah River Company Savannah River National Laboratory Aiken, SC 29808

Prepared for the U.S. Department of Energy Under Contract No. DE-AC09-96SR18500

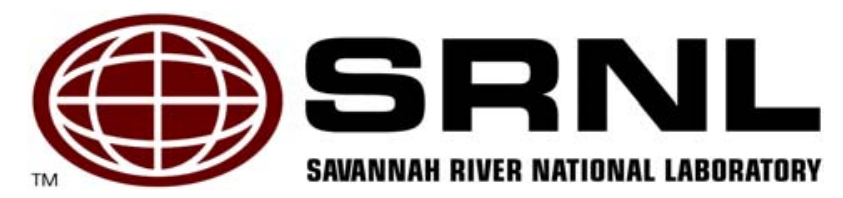




\section{DISCLAIMER}

This report was prepared by Washington Savannah River Company (WSRC) for the United States Department of Energy under Contract No. DE-AC09-96SR18500 and is an account of work performed under that contract. Neither the United States Department of Energy, nor WSRC, nor any of their employees makes any warranty, expressed or implied, or assumes any legal liability or responsibility for the accuracy, completeness, or usefulness, of any information, apparatus, or product or process disclosed herein or represents that its use will not infringe privately owned rights. Reference herein to any specific commercial product, process, or service by trademark, name, manufacturer or otherwise does not necessarily constitute or imply endorsement, recommendation, or favoring of same by WSRC or by the United States Government or any agency thereof. The views and opinions of the authors expressed herein do not necessarily state or reflect those of the United States Government or any agency thereof.

\section{Printed in the United States of America}

Prepared For

\section{U.S. Department of Energy}


Keywords: Particle collection, Acoustic force Aerosol flow, Turbulent flow, Computational Fluid Dynamics

\section{Evaluation of Acoustic Forces on A Particle in Aerosol Medium}

Richard A. Dimenna and Si Young Lee

September 2007

Washington Savannah River Company Savannah River National Laboratory Aiken, SC 29808

Prepared for the U.S. Department of Energy Under Contract No. DE-AC09-96SR18500

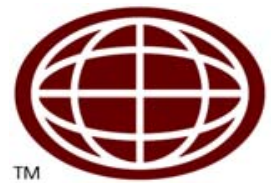




\section{Review and Approvals}

R. A. Dimenna, Author Date

Eng. Modeling and Simulation Groun. SRNL

S. Y. Lee, Coauthor

Date

Enq. Modelina and Simulation Groun SRNI

B. B. Anderson, Customer Reviewer

Date

Nonproliferation Tech. Section, SRNL

IVI. v. Gregory, I ecnnıcal Reviewer

Date

Eng. Modeling and Simulation Group, SRNL

S. J. Hensel, Manager

Date

Eng. Modeling and Simulation Group, SRNL 


\section{Table of Contents}

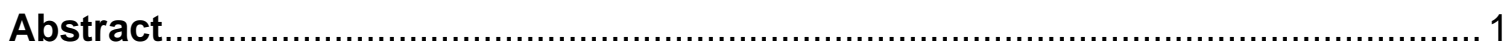

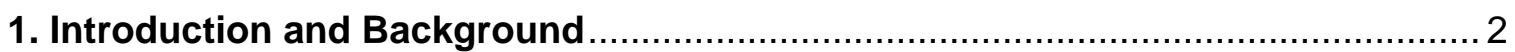

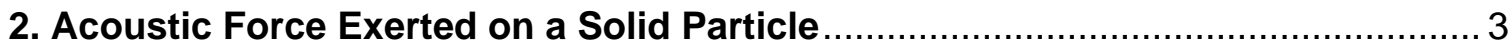

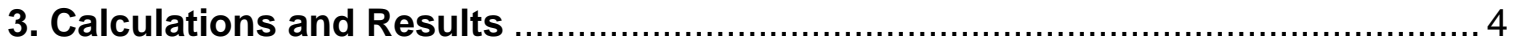

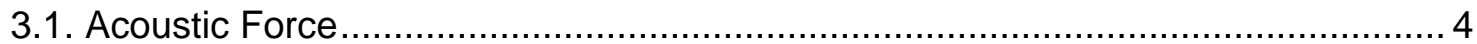

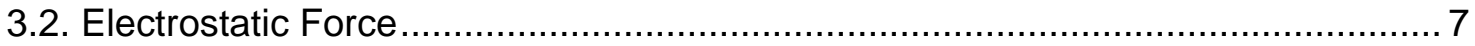

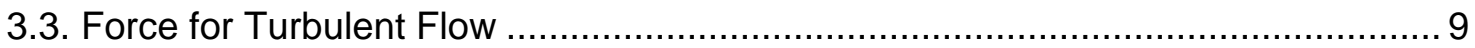

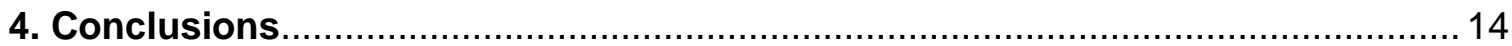

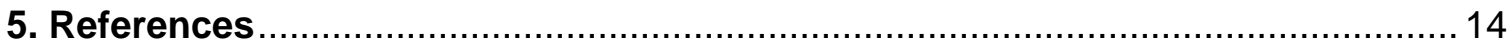




\section{List of Figures}

Figure 1. Standing acoustic wave profile and acoustic force for particle $\left(F_{a}\right)$ in a rectangular flow channel laden with particles ............................................. 2

Figure 2. Acoustic force on particle for various sizes of particles ................................ 6

Figure 3. Cross-sectional top view of the SRNL test section with electrostatic field assuming zero aerosol flow $(\mathrm{L}=1$ inch, Voltage $=18 \mathrm{kV}) \ldots \ldots \ldots \ldots \ldots \ldots \ldots \ldots \ldots \ldots . . .1$

Figure 4. Comparison of electrostatic forces and acoustic forces with an electrostatic field as shown in Fig. 3 ....................................................................... 8

Figure 5. Comparison of three different forces as shown in Fig. 1............................. 10

Figure 7. Acoustic forces for different wavelengths under $195 \mathrm{~dB}$ acoustic wave and 1-

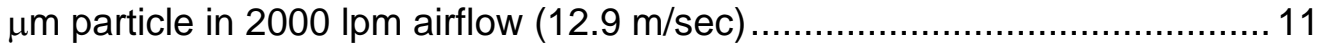

Figure 8. Acoustic forces on 1- $\mu \mathrm{m}$ particle in $2000 \mathrm{lpm}$ airflow $(12.9 \mathrm{~m} / \mathrm{sec})$ for different wavelengths under different acoustic pressure amplitudes

Figure 9. Comparison of various forces as function of particle sizes in $2000 \mathrm{Ipm}$ airflow $(12.9 \mathrm{~m} / \mathrm{sec})$

\section{List of Tables}

Table 1. Material properties and conditions used for the present analysis 5

Table 2. Acoustic power generation per unit area under various sound pressure levels. 6

Table 3. Comparison of three major forces under three different particle diameters 13 


\section{Abstract}

The acoustic force exerted on a solid particle was evaluated to develop a fundamental understanding of the critical physical parameters or constraints affecting particle motion and capture in a collecting device. The application of an acoustic force to the collection of a range of submicron-to-micron particles in a highly turbulent airflow stream laden with solid particles was evaluated in the presence of other assisting and competing forces. This scoping estimate was based on the primary acoustic force acting directly on particles in a dilute aerosol system, neglecting secondary interparticle effects such as agglomeration of the sub-micron particles.

A simplified analysis assuming a stable acoustic equilibrium with an infinite sound speed in the solid shows that for a solid-laden air flow in the presence of a standing wave, particles will move toward the nearest node. The results also show that the turbulent drag force on a 1- $\mu \mathrm{m}$ particle resulting from eddy motion is dominant when compared with the electrostatic force or the ultrasonic acoustic force. At least $180 \mathrm{~dB}$ acoustic pressure level at $1 \mathrm{MHz}$ is required for the acoustic force to be comparable to the electrostatic or turbulent drag forces in a high-speed air stream.

It is noted that particle size and pressure amplitude are dominant parameters for the acoustic force. When acoustic pressure level becomes very large, the acoustic energy will heat up the surrounding air medium, which may cause air to expand. With an acoustic power of about 600 watts applied to a 2000-Ipm air flow, the air temperature can increase by as much as $15^{\circ} \mathrm{C}$ at the exit of the collector. 


\section{Introduction and Background}

High-frequency sound waves have been widely explored as a means of causing agglomeration or coalescence of particles and thereby promoting their ease of separation from a gas stream. Though not found to be widely practical in commercial application, sonic collectors have been used successfully in certain dust-removal operations. Within a vibrating sonic field under turbulent airflow, there are three modes of force acting on a suspended particle: Bernoulli forces within the medium between particles; shear forces on the particle surface due to bulk motion and eddy-induced fluctuations within medium; and sound-wave radiation pressure owing to induced momentum differences on either side of the vibrating particle. Counteracting or overshadowing these forces are the effects of Brownian movement and gravitational free-fall velocity. Eddy motions due to turbulent-induced secondary flow may also cause a counteractive force against the acoustic compressive force associated with particle agglomeration.

The primary objective of this work is to quantify the acoustic forces exerted on a solid particle to obtain a fundamental understanding of critical physical parameters or constraints, and to explore their acoustic applications to the collection of submicron-tomicron particles under the influence of a standing acoustic wave as sketched in Fig. 1. Attention is focused on the quantitative comparison of acoustic force with other surface forces such as Coulomb force due to electrostatic surface charge and hydrodynamic drag force on solid surface due to the surrounding turbulent airflow. The magnitude of the electrostatic force and the surface drag force due to turbulent eddies has been also evaluated.

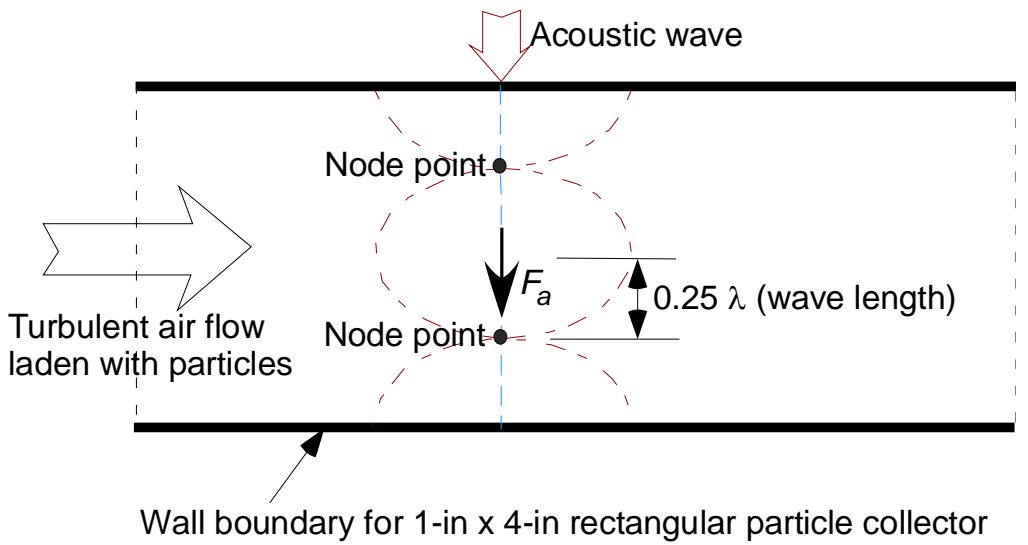

Figure 1. Standing acoustic wave profile and acoustic force for particle $\left(F_{a}\right)$ in a rectangular flow channel laden with particles 


\section{Acoustic Force Exerted on a Solid Particle}

The present work considered the time-averaged acoustic wave force exerted on a spherical solid in an acoustic standing wave. If the particles carried by gas flow are exposed to an ultrasonic field as shown in Fig. 1, they experience pressure gradients due to the acoustic standing wave. These pressure differences arise from the displacement of the molecules of the medium. The acoustic pressure gradients give rise to acoustic forces. Their magnitude and direction depend on the difference in the mechanical properties between the particles and the host medium. The mechanical properties closely related to the acoustic force are density and compressibility.

The ultrasonic acoustic wave will give rise to two types of acoustic forces. One is primary acoustic forces that act directly on particles, and the other is secondary acoustic forces that act between the particles causing attraction or repulsion. These interparticle forces tend to drive particles together so that tiny particles become big particles. The force can grow rapidly since the acoustic force is proportional to particle volume. In this work, the secondary forces due to interparticle effects are assumed to be negligible because the solids loading of the air flow is small.

When the ultrasonic field is in the form of a standing wave, the acoustic pressure $[1,6]$ can be written as

$$
\begin{aligned}
P & =P_{o} \sin (k x) \cos (\omega t) \\
& =P_{o} \sin \left(\frac{2 \pi}{\lambda} x\right) \cos \left(\frac{2 \pi}{T} t\right)
\end{aligned}
$$

In eq. (1) $P_{o}$ is the pressure amplitude, $k$ wave number, $\lambda$ wavelength, $T$ period, $\mathrm{t}$ time, $\mathrm{x}$ local position, and $\omega$ angular frequency, respectively. According to the acoustic force theory presented by Yosioka and Kawasima [1], the force on a particle due to an acoustic wave, $F_{a}$, can be expressed as

$$
F_{a}=-\left(\frac{\pi P_{o}^{2} V_{p} \beta_{f}}{2 \lambda}\right) \zeta \sin (2 k x)
$$

In eq. (2) the parameters, $V_{p}, \beta_{f}$, and $\zeta$, are the volume of particle, fluid compressibility and the material constant related to density and compressibility, respectively. When the constant $\zeta$ is positive, the force will pull the particle toward the node, and when it becomes opposite in sign, it will move the particle toward the anti-node. The material constant $\zeta$ is obtained by the following expression [6]:

$\zeta=\left(\frac{5 \rho_{p}-2 \rho_{f}}{2 \rho_{p}+\rho_{f}}\right)-\left(\frac{\beta_{p}}{\beta_{f}}\right)$

In eq. (3) $\beta_{f}$ and $\beta_{p}$ are material compressibilities for the fluid medium and particle, respectively. In this case, $\beta_{p}$ are essentially zero because of the assumption of an incompressible solid material. Thus, the acoustic force always pulls the particles toward the node, since the $\zeta$ term in eq. (3) is always positive. Air compressibility, $\beta_{f}$,can be related to the sound speed $c_{f}$. [2]

$$
\beta_{f}=\left(\frac{1}{\rho_{f} c_{f}^{2}}\right)
$$


The acoustic wavelength $\lambda$ can be expressed in terms of the sound speed, $c_{f}$, and the frequency $f$.

$$
\lambda=\left(\frac{c_{f}}{f}\right)
$$

By substituting eqs. (4) and (5) into eq. (2), the maximum acoustic force $F_{a, \max }$ acting on particle volume $V_{p}$ can be expressed in terms of the frequency $f$.

$$
F_{a, \max }=\left(\frac{\pi P_{o}^{2} V_{p}}{2 \rho_{f} c_{f}^{3}}\right)\left(\frac{5 \delta-2}{2 \delta+1}\right) f=\left(\frac{\pi P_{o}^{2} V_{p}}{2 \rho_{f} c_{f}^{3}}\right) \xi f
$$

In eq. (6), $\delta$ and $\xi$ are the density ratio of the particle to the fluid medium and the material constant, respectively.

\section{Calculations and Results}

When the air medium has a dilute particle concentration in a stationary sound field, the maximum acoustic force exerted on a solid sphere of diameter $d_{p}$ is provided by eq. (6). As seen in the equation, the acoustic radiation force acting on a solid particle in the standing wave field is primarily dependent on particle size and pressure amplitude. It is noted that the acoustic force on a solid particle in an air medium is maximum halfway between the acoustic wave nodes and anti-nodes and inversely proportional to the acoustic wavelength.

In this work, one of the main goals was to make a quantitative comparison of the acoustic force with other potential surface forces such as the Coulomb force due to the electrostatic surface charge and the hydrodynamic drag force on solid surface in a fast flowing gas stream. The magnitudes of the electrostatic and surface drag forces due to turbulent eddies are evaluated.

\subsection{Acoustic Force}

As discussed earlier, with a density ratio $\delta$ of about 1265 under the modeling conditions in Table 1, the material constant $\xi$ is always positive and so is the maximum acoustic force $F_{a, \max }$. Thus, particle agglomeration is toward the nearest node at stable acoustic equilibrium. It is important to have a strong force to improve the particle collection efficiency in a high speed air flow environment. According to the governing equation for acoustic force, the force is rapidly increased with the sound pressure level.

A sound pressure level is measured in decibels $(\mathrm{dB})$, where $0 \mathrm{~dB}$ is the reference to the threshold of human hearing. A pressure level of $1 \mathrm{~Pa}$ is equal to $94 \mathrm{~dB}$. When a sound is created for an engineering application of acoustic force, it is important to measure the distance from the source, since the acoustic pressure level is inversely proportional to the distance from a point source. Hence, acoustic pressure $(p)$ is related to acoustic power per unit area or acoustic intensity $(I)$ in watts $/ \mathrm{m}^{2}$ or watts/inch ${ }^{2}$ by the acoustic impedance $(Z)$ in $\mathrm{Pa}-\mathrm{sec} / \mathrm{m}$ in an air medium. The impedance, $Z$, is the product of the sound speed and the host medium density [3]. That is,

$p=\sqrt{I Z}=\sqrt{I c_{f} \rho_{f}}$ 
For an isotropic, spherical sound source, the acoustic intensity $(I)$ at position $r$ along the radial direction from the center of the source is equal to total power divided by the spherical surface area, $4 \pi r^{2}$. Table 2 shows the acoustic intensity from a sound pressure level in a $25^{\circ} \mathrm{C}$ air medium. As shown in the table, the increased power will heat up the surrounding air due to dissipation of the acoustic energy, which may cause the air to expand. When an acoustic power of about 600 watts generated by a $180 \mathrm{~dB}$ acoustic pressure level is applied to 2000-Ipm air flow, the air temperature will increase about $15{ }^{\circ} \mathrm{C}$ at exit of the collector. This estimate is based on an assumption of an adiabatic wall boundary for the collector system.

Based on material properties and geometrical conditions as provided in Table 1, acoustic forces on a spherical particle for a range of sizes are presented in Fig. 2. The results show that when the acoustic pressure amplitude is increased from $180 \mathrm{~dB}$ to 190 $\mathrm{dB}$ at $1 \mathrm{MHz}$, the acoustic force on a $1-\mu \mathrm{m}$ particle increases 0.02 to $0.2 \mathrm{nN}\left(10^{-9} \mathrm{~N}\right)$. It is noted that particle size and pressure amplitude are dominant factors for the acoustic force on the solid particle. The theoretical limit of acoustic pressure level for a sound wave at $1 \mathrm{~atm}$ pressure is about $194 \mathrm{~dB}$, which corresponds to generation of $0.1 \mathrm{MPa}$ compression pressure. [3]

Table 1. Material properties and conditions used for the present analysis

\begin{tabular}{|c|c|}
\hline Parameters & Values \\
\hline Geometry and dimensions & Rectangular channel ( 1 in high and 4 in wide) \\
\hline Air flowrate (liters/min.) & 2,000 \\
\hline Air inlet velocity (m/sec) & 12.9 \\
\hline Air density (kg/m $\left.{ }^{3}\right)$ & 1.225 \\
\hline Particle density $\left(\mathrm{kg} / \mathrm{m}^{3}\right)$ & 1,550 \\
\hline $\begin{array}{c}\text { Sound wave frequency (MHz) } \\
\text { Sound speed in air (m/sec) }\end{array}$ & 340 \\
\hline $\begin{array}{c}\text { Range of particle diameters } \\
\text { (micrometers) }\end{array}$ & 0.5 to 5 \\
\hline
\end{tabular}


Table 2. Acoustic power generation per unit area under various sound pressure levels

\begin{tabular}{|c|c|c|}
\hline $\begin{array}{c}\text { Sound pressure } \\
\text { level (dB) }\end{array}$ & $\begin{array}{c}\text { Acoustic pressure } \\
\text { (Pa) }\end{array}$ & $\begin{array}{c}\text { Acoustic intensity } \\
\text { (watts/inch }^{2} \text { ) }\end{array}$ \\
\hline 160 & 2000 & 6.1 \\
\hline 170 & 6325 & 60.9 \\
\hline 180 & 20000 & 608.9 \\
\hline 190 & 63246 & 6088.6 \\
\hline
\end{tabular}

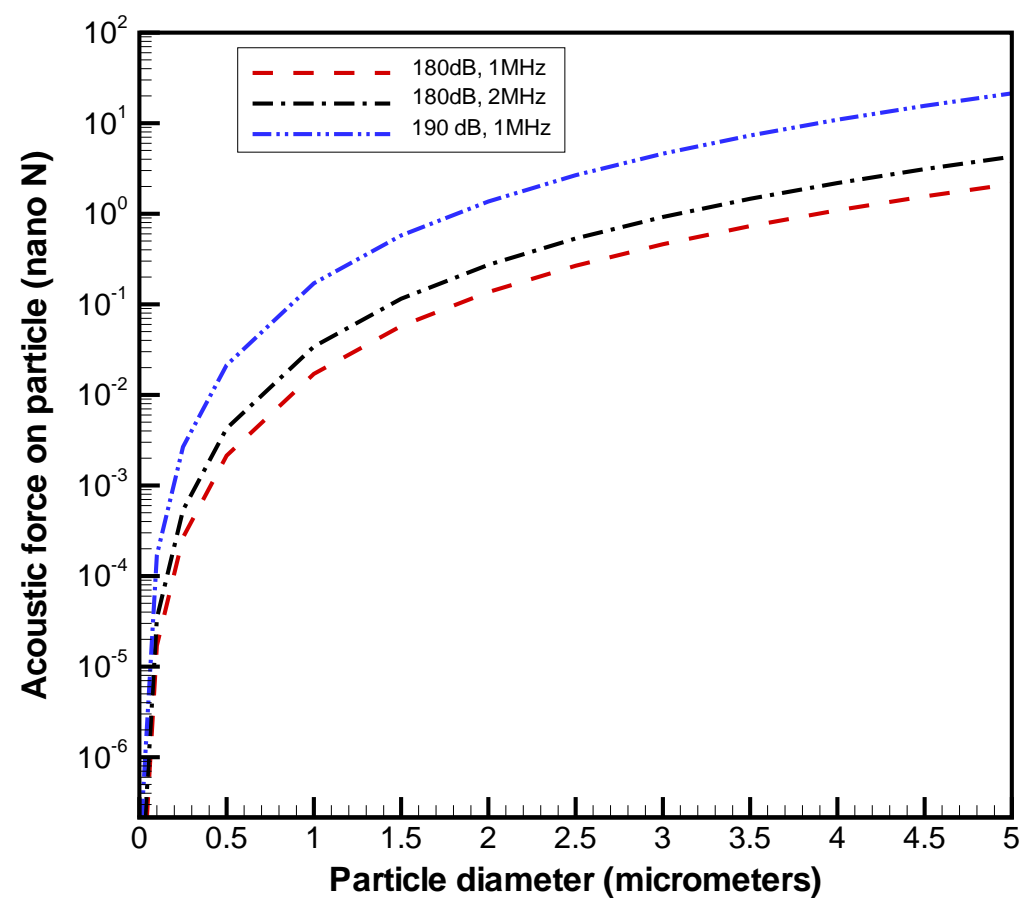

Figure 2. Acoustic force on particle for various sizes of particles

\subsection{Electrostatic Force}

Figure 3 schematically illustrates a cross-sectional top view of the SRNL test section with an electrostatic field assuming zero aerosol flow. 


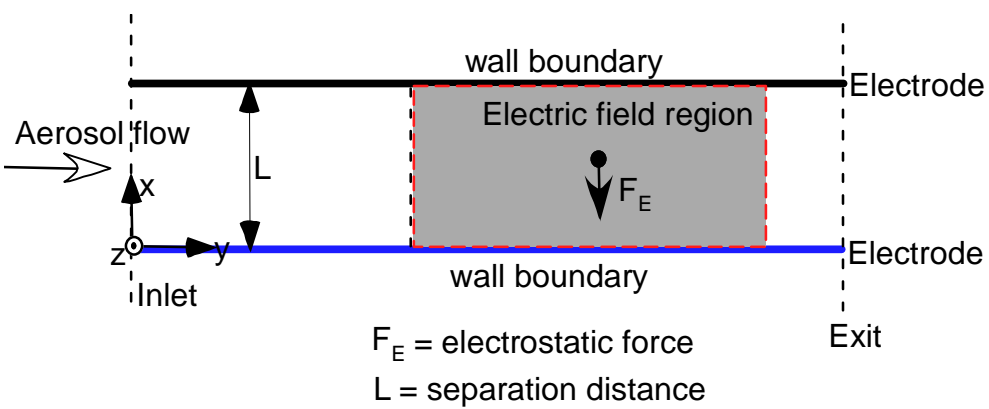

Figure 3. Cross-sectional top view of the SRNL test section with electrostatic field assuming zero aerosol flow $(\mathrm{L}=1$ inch, Voltage $=18 \mathrm{kV})$

When a charged particle is suspended in a gas near a conducting surface, the electrostatic force $F_{E}$ shown in Fig. 3 is governed by the equation $[8,11]$ :

$$
F_{E}=q E-\frac{q^{2}}{16 \pi \varepsilon_{0} x^{2}}+\frac{q E d^{3}}{16 x^{3}}-\frac{3 \pi \varepsilon_{0} d^{6} E^{2}}{128 x^{4}}
$$

and

$E=\frac{V}{L}$,

where $L$ is a separation distance perpendicular to the flow direction (y-direction).

The first term is the Coulomb force due to the applied electric field. The second term on the right-hand side is the image force corresponding to the force exerted by an image charge of $-q$ at position $-x$ from the surface. The third term is the dielectrophoretic force on the induced dipole due to the gradient of the field from the image charge. The last term is the dipole-dipole force due to the interaction of the induced dipole and its image. The image and dipole-dipole forces are always directed toward the surface, while the Coulomb and dielectrophoretic forces can be either toward or away from the wall. In this study, Coulomb and image forces are included in the analysis since the scoping calculations show that the other forces are negligibly small.

Usually the charging process is divided into a field charging region for particles greater than $0.1 \mu \mathrm{m}$ and a diffusion charging region for particles less than $0.1 \mu \mathrm{m}$. While field charging requires the presence of an electric field which drives the free movable charge carriers, the diffusion process is based on randomly moving gas ions caused by temperature and described by kinetic gas theory and phenomena such as Brownian motion.

The particle saturation charge after infinite time is given by Cochet's equation [8]. According to his equation, particle charge $q$ is a function of particle size and can be described by Cochet's charge equation.

$$
q=4 \pi \varepsilon_{o} \operatorname{Er}^{2}\left[\left(1+\frac{\lambda_{i}}{r}\right)^{2}+\left(\frac{2}{1+\left(\frac{\lambda_{i}}{r}\right)}\right)\left(\frac{\kappa-1}{\kappa+2}\right)\right]
$$


where $\varepsilon_{0}$ is the permittivity of free space $\left(8.85 \times 10^{-12} \mathrm{~N} / \mathrm{m}\right), E$ the local electric field strength, $\lambda_{i}$ the ionic mean free path, $r$ the particle radius, and $\kappa$ the dielectric constant of particles. Typical conditions of $\lambda_{i}=0.065$ to $0.1 \mu \mathrm{m}$ and $\kappa=10$ were applied here. Figure 4 presents comparison of electrostatic forces and acoustic forces with an electrostatic field as shown in Fig. 3. The results show that an acoustic pressure level of about $185 \mathrm{~dB}$ is comparable to the electrostatic force due to saturated charging of submicron particles from an $18 \mathrm{kV}$ electrical potential. It is noted that for less than 170 $\mathrm{dB}$ acoustic level, the electrostatic force is much higher than the acoustic force.

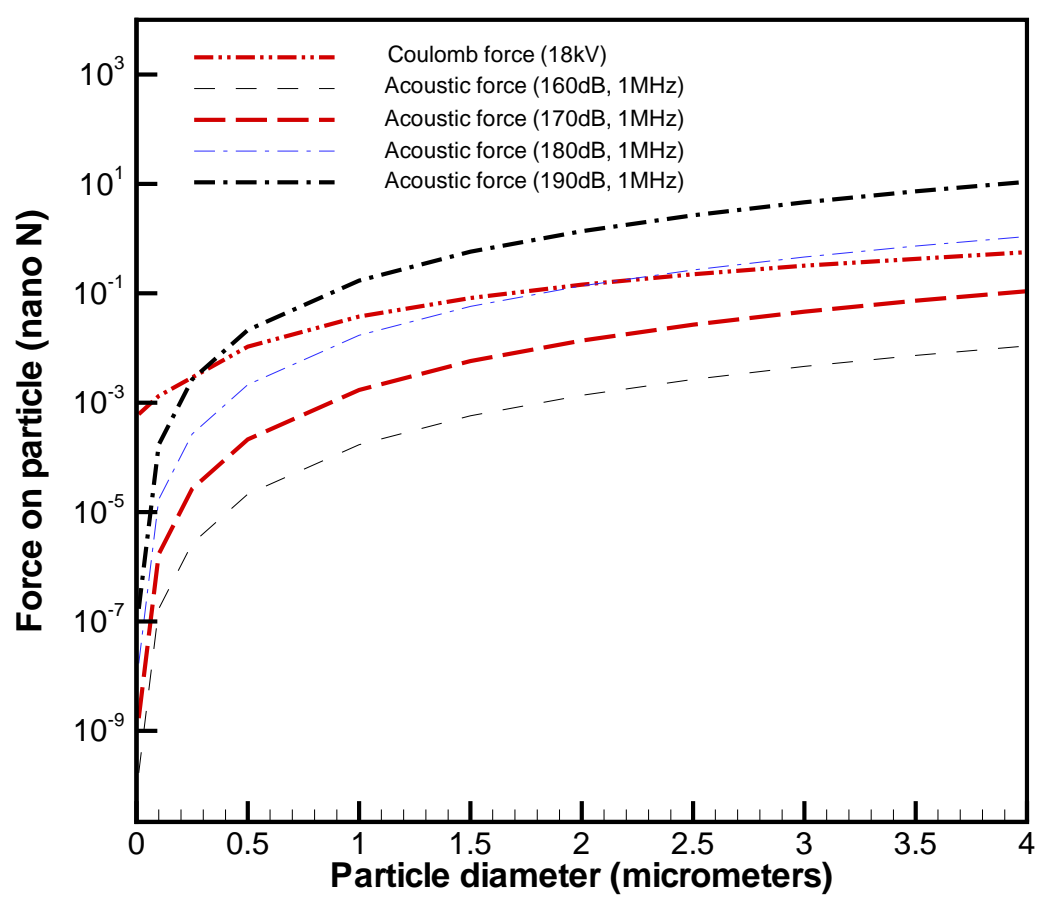

Figure 4. Comparison of electrostatic forces and acoustic forces with an electrostatic field as shown in Fig. 3

\subsection{Force for Turbulent Flow}

When air flows at 2,000 lpm through the rectangular collector evaluated in this work, the flow is fully-turbulent, since the particle Reynolds number is about 2,000 in based on a 1$\mu \mathrm{m}$ particle diameter. The force due to turbulent shear is given in terms of the mean velocity magnitude $(U)$

$$
F_{\text {turb }}=\frac{1}{2} C_{d} \rho_{f} U^{2} A_{p}=\frac{\pi}{8} C_{d} \rho_{f} U^{2} d_{p}^{2}
$$


In eq. (11), $C_{d}$ and $d_{p}$ are the drag coefficient and particle diameter, respectively. From the literature, the drag coefficient $C_{d}$ is about 0.6 for a spherical particle in turbulent flow [9]. Table 3 shows a quantitative comparison of three major surface forces for different particle sizes with an $18 \mathrm{kV}$ potential difference across the collection region, an acoustic standing wave, and turbulent drag due to shear. Figure 5 compares the surface forces for a range of particle sizes.

For very dilute particle concentrations, the character of particle motion depends on its size relative to the scale of turbulence of the flow. When the particle size is small compared with the eddy scale of turbulent flow, its motion is greatly affected by fluctuations in the local velocity, and the particle does not act as a disturbing element in the flow field. Depending on its size and the relative density, it may well respond to some of the faster, smaller-scale fluid motions. As illustrated in Fig. 6, turbulent flow eddies of different sizes are imposed onto the mean flow. When a small particle enters the turbulent region, it follows a path dictated by both the mean bulk flow (streamlines) and the eddies. Larger eddies carry the particle laterally across streamlines, while smaller eddies create smaller scale stirring that causes the particles to spread or diffuse. Thus, turbulent eddies create fluctuations in velocity. Velocity fluctuation magnitudes vary in time due to continuous eddy generation, leading to the turbulent dissipation of fluid momentum. The measured velocity $(v)$ includes both the mean velocity magnitude $(U)$ and the fluctuation component $(u)$.

$v=U+u$

In eq. (12), the fluctuation velocity is expressed as the root-mean-square (rms) value of the set of random velocity fluctuations. In evaluating the effect of turbulence on a particle in a very dilute system, assumptions were made to calculate a force due to the velocity fluctuations. The particle is small enough so that its motion relative to the fluid can be given by Stoke's drag. For a 1- $\mu \mathrm{m}$ particle traveling in a $2000 \mathrm{lpm}$ and a $1 \times 4$ in rectangular channel, Reynolds number for turbulent eddy motion is about 0.1 . This is Stoke's regime according to the literature [10]. In this case, the drag coefficient $\left(C_{d}\right)$ on the spherical surface of a solid particle with diameter $d_{p}$ is given by

$C_{d}=\frac{24}{\operatorname{Re}_{p}}=\frac{24 \mu}{d_{p} \rho u}$

In eq. (13), $\mu$ and $u$ parameters are fluid viscosity and magnitude of velocity fluctuation, respectively. When turbulent kinetic energy is associated with momentum dissipation, the rms value of the velocity fluctuation is generally estimated at about $10 \%$ of the mean bulk velocity magnitude for a fully turbulent flow condition [12]. Thus, the turbulent fluctuation force on the particle due to the turbulent eddy can be estimated by eqs. (11) and (13). The results show that when the particle diameter is less than $1 \mu \mathrm{m}$ and interparticle interactions are negligible, the turbulent fluctuation force has a large impact on particle motion. In this case, solid particles are essentially controlled by fluid eddy motions.

Figure 7 compares electrostatic and turbulent forces with the acoustic forces with different wavelengths under $195 \mathrm{~dB}$ acoustic level exerted on a 1- $\mu \mathrm{m}$ particle under 2000 Ipm airflow $(12.9 \mathrm{~m} / \mathrm{sec})$. Acoustic forces on a 1- $\mu \mathrm{m}$ particle for different wavelengths under different acoustic pressure amplitudes are compared with other forces in $2000 \mathrm{lpm}$ 
airflow $(12.9 \mathrm{~m} / \mathrm{sec})$ in Fig. 8. Figure 9 makes a quantitative comparison of three major forces exerted on solid particles of various particle sizes in $2000 \mathrm{lpm}$ airflow.

The results clearly show that turbulent drag forces on a 1- $\mu \mathrm{m}$ particle due to bulk shear and eddy motion are dominant when compared with electrostatic and ultrasonic forces. Table 3 compares the calculated results for the forces for three different particle sizes.

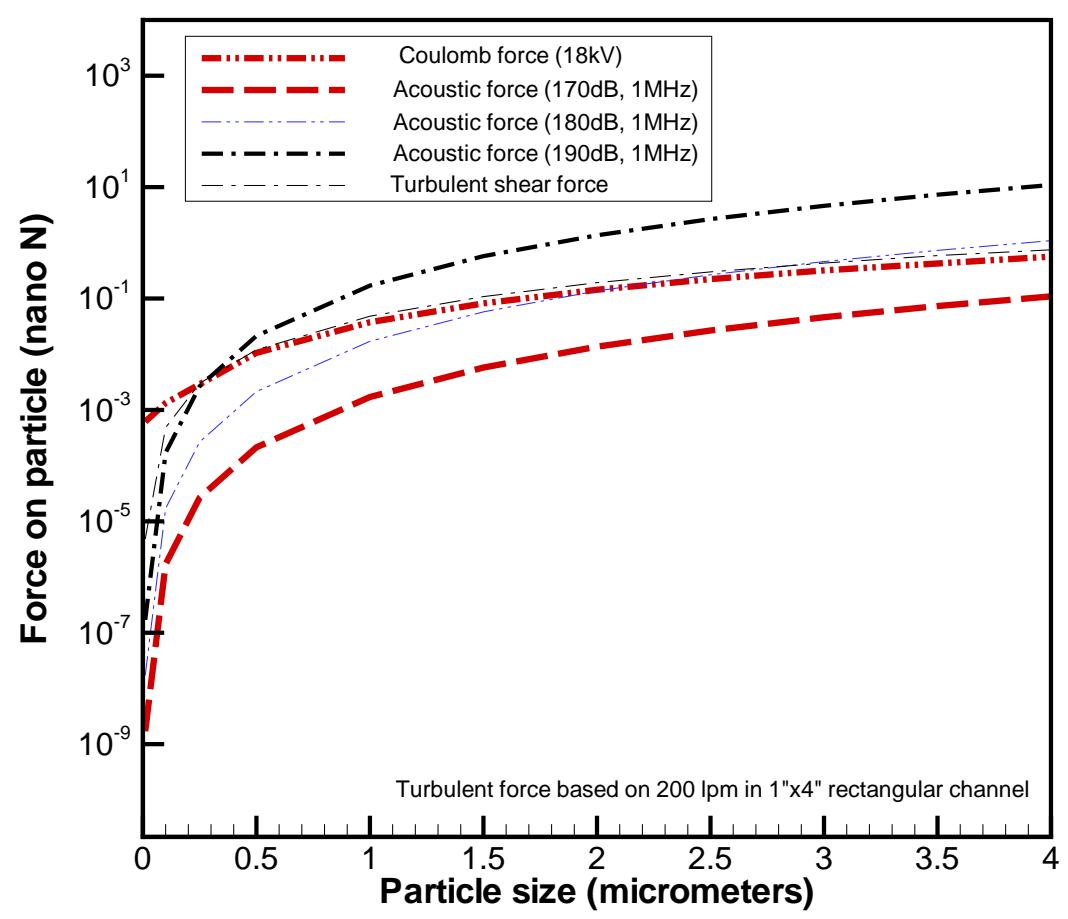

Figure 5. Comparison of three different forces as shown in Fig. 1

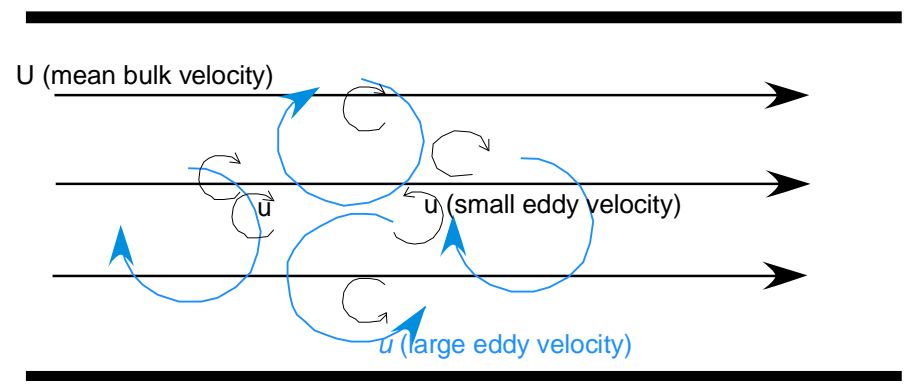

Figure 6. Particle movement in turbulent flow with large and small flow eddies 

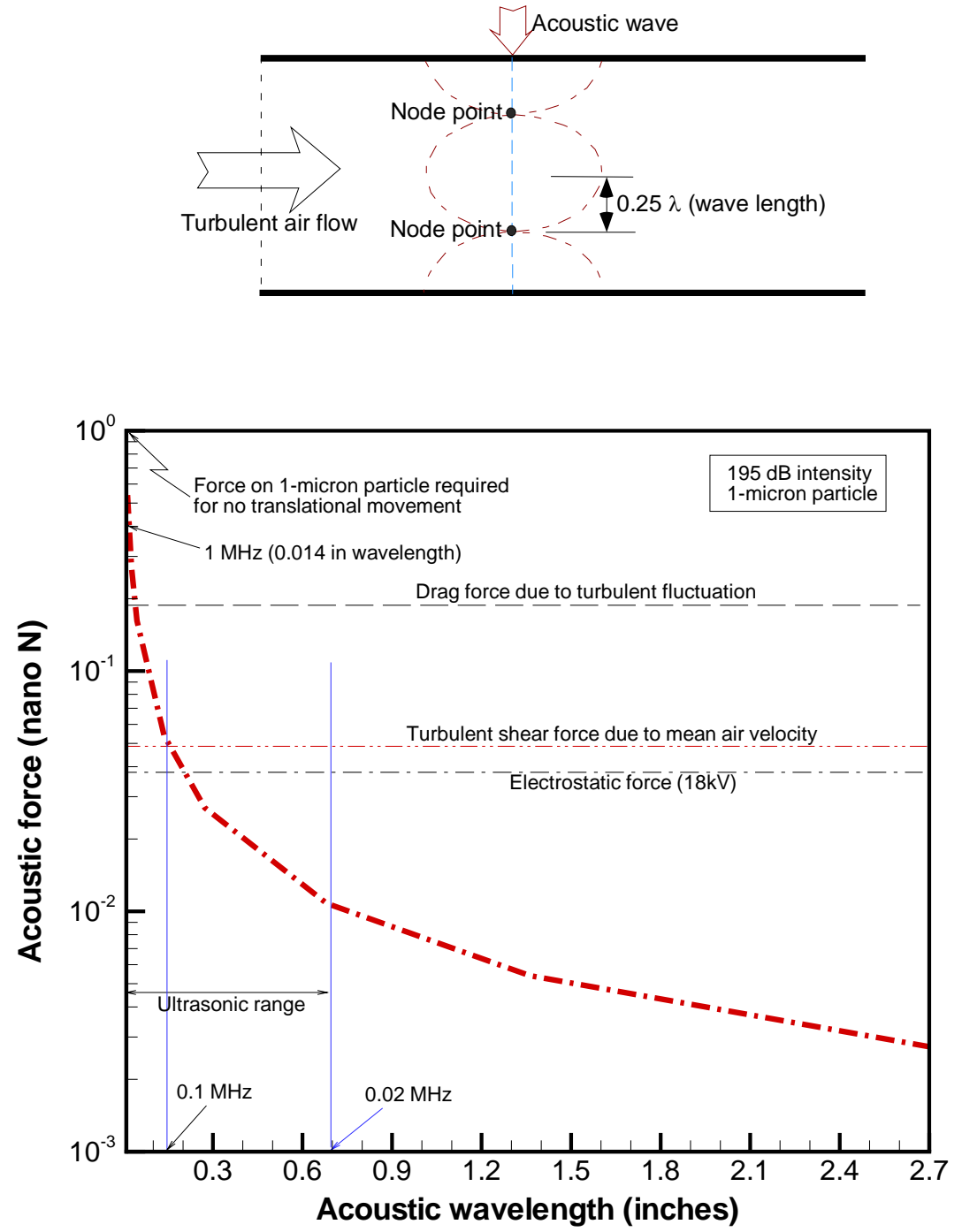

Figure 7. Acoustic forces for different wavelengths under $195 \mathrm{~dB}$ acoustic wave and 1$\mu \mathrm{m}$ particle in $2000 \mathrm{lpm}$ airflow $(12.9 \mathrm{~m} / \mathrm{sec})$ 


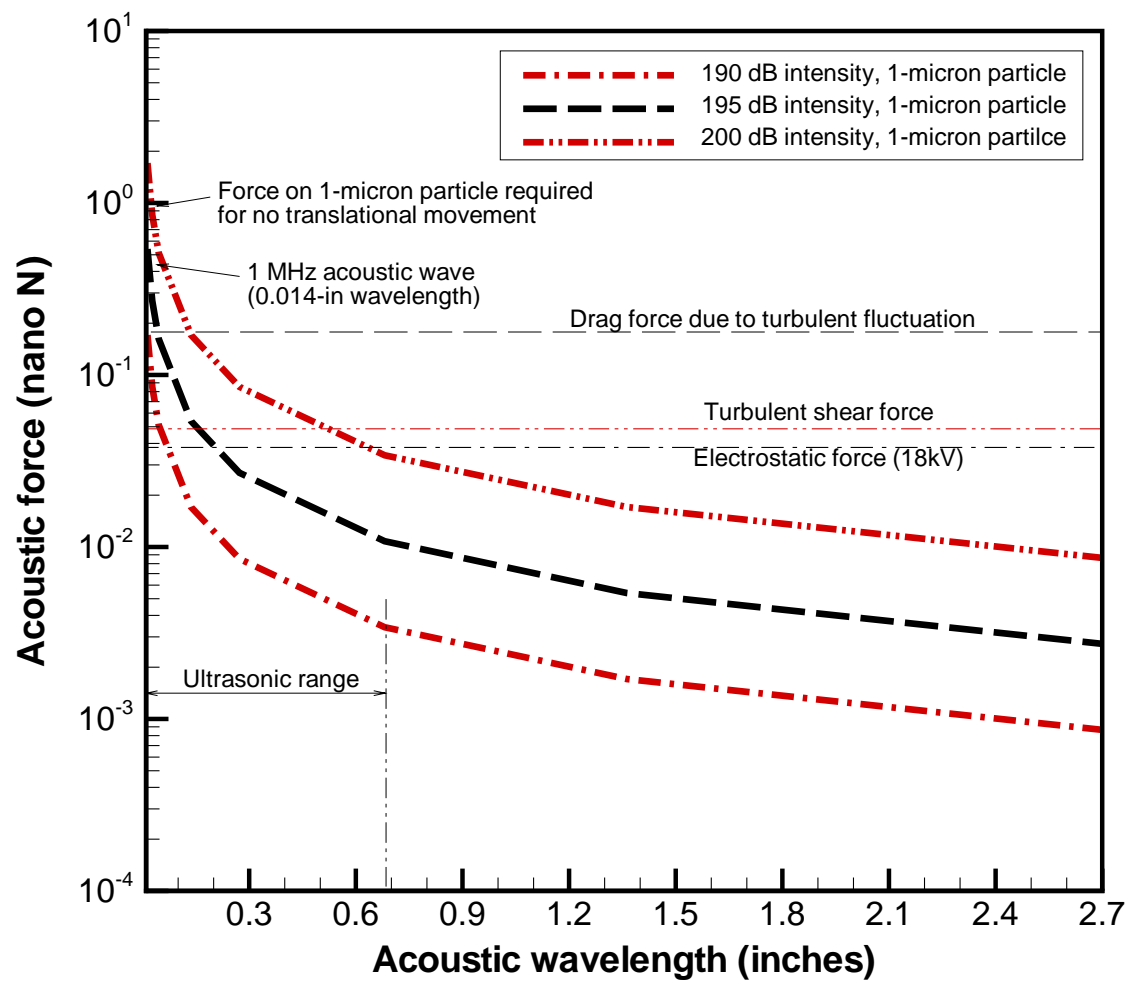

Figure 8. Acoustic forces on 1- $\mu \mathrm{m}$ particle in $2000 \mathrm{lpm}$ airflow $(12.9 \mathrm{~m} / \mathrm{sec})$ for different wavelengths under different acoustic pressure amplitudes 


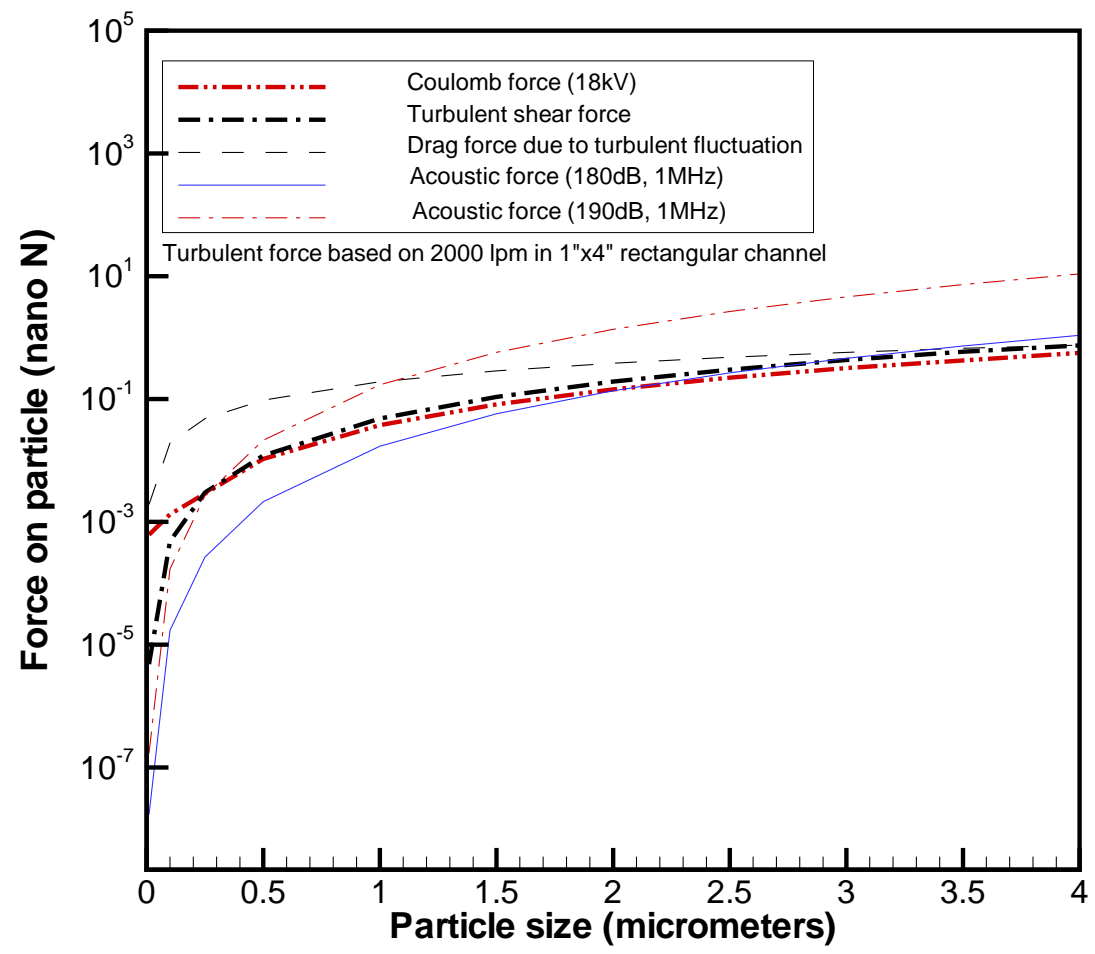

Figure 9. Comparison of various forces as function of particle sizes in 2000 lpm airflow $(12.9 \mathrm{~m} / \mathrm{sec})$

Table 3. Comparison of three major forces under three different particle diameters

\begin{tabular}{|c|c|c|c|c|c|}
\hline \multirow{2}{*}{$\begin{array}{c}\text { Particle diameter } \\
\text { (micrometer) }\end{array}$} & $\begin{array}{c}\text { Electrostatic } \\
\text { force, nanoN }\end{array}$ & \multicolumn{2}{|c|}{$\begin{array}{c}\text { Acoustic force } \\
\text { nanoN }\end{array}$} & \multicolumn{2}{c|}{ Turbulent force*, nanoN } \\
\cline { 3 - 6 } & $\begin{array}{c}180 \mathrm{~dB}, \\
1 \mathrm{MHz}\end{array}$ & $\begin{array}{c}190 \mathrm{~dB}, \\
1 \mathrm{MHz}\end{array}$ & $\begin{array}{c}\text { Turbulent } \\
\text { shear }\end{array}$ & $\begin{array}{c}\text { Turbulent } \\
\text { fluctuation }\end{array}$ \\
\hline 0.5 & 0.011 & 0.002 & 0.021 & 0.012 & 0.096 \\
\hline 1 & 0.038 & 0.017 & 0.171 & 0.048 & 0.192 \\
\hline 2 & 0.144 & 0.137 & 1.366 & 0.193 & 0.383 \\
\hline
\end{tabular}

Note:*Based on local velocity corresponding to air flowrate of 2,000 liter/min in 1 in $\times 4$ in rectangular channel 


\section{Conclusions}

The acoustic forces exerted on a solid particle were estimated to obtain a fundamental understanding of the critical physical parameters or constraints and to explore acoustic applications for collecting of a range of submicron-to-micron particles under the influence of a standing acoustic wave. This estimate was based on the primary acoustic force acting directly on particles, neglecting the secondary interparticle effects such as agglomeration of sub-micron particles.

The main conclusions are as follows:

- Acoustic forces for various sound levels were compared with other surface forces such as Coulomb force due to an electrostatic surface charge and the hydrodynamic drag force on the solid particle surface due to turbulent airflow.

- When the acoustic force exerted on a micron-size particle in a high-speed air stream is considered, at least $180 \mathrm{~dB}$ acoustic pressure level is required to be comparable to the electrostatic and turbulent forces

- The results show that turbulent drag forces on a 1- $\mu \mathrm{m}$ particle due to bulk shear and eddy motion are dominant when compared with electrostatic and ultrasonic forces.

- For a high level of the acoustic pressure, the increased acoustic intensity will heat the surrounding air, which may cause air to expand. When an acoustic power of about 600 watts generated by $180 \mathrm{~dB}$ acoustic pressure level is applied to the 2000-Ipm SRNL particle collector, the air temperature will increase by as much as $15^{\circ} \mathrm{C}$ at exit of the collector.

\section{References}

1. K. Yosiok and Y. Kawasima, "Acoustic Radiation Pressure on a Compressible Sphere", Acustica, Vol. 5, pp. 167-173, 1955.

2. L. L. Beranek, Acoustics, Acoustic Society of America, 1993.

3. C. L. Morfey, Dictionary of Acoustics, Academic Press, San Diego, 2001.

4. W. C. Hinds, "Aerosol Technology Properties, Behavior, and Measurement of Airborne Particles," $2^{\text {nd }}$ Edition, John Wiley \& Sons, Inc., 1999.

5. H. M. Hertz, "Standing Wave Acoustic Trap for Nonintrusive Positioning of Microparticles", J. of Applied Physics, Vol. 78, pp. 4545-4849, 1995.

6. L. A. Cruz, "Acoustic Force on a Liquid Droplet in an Acoustic Stationary Wave", J. of the Acoustical Society of America, Vol. 50, pp. 157-163, 1971.

7. D. W. Cooper, M. H. Peters, and R. J. Miller, "Predicted Deposition of Submicron Particles due to Diffusion and Electrostatics in Viscous Axisymmetric StagnationPoint Flow", Aerosol Science Technology Vol. 11, pp. 133-143, 1989.

8. R. Cochet, "Lois Charge des Fines Particules (Submicroniques) Etudes Theoriques - Controles Recents Spectre de Particules", Coll. Int. la Physique des Forces Electrostatiques et Leurs Application, Centre National de la Recherche Scientifique, Paris, Vol. 102, pp. 331-338, 1961.

9. V. L. Streeter, Fluid Mechanics, McGraw-Hill, Inc., 1951. 
10. R. B. Bird, W. E. Stewart, and E. N. Lightfoot, Transport Phenomena, John Wiley \& Sons, 1960.

11. A. Li and G. Ahmadi, "Aerosol Particle Deposition with Electrostatic Attraction in a Turbulent Channel Flow", J. of Colloid and Interface Science, Vol. 158, pp. 476482, 1993.

12. H. Tennekes and J. L. Lumley, A First Course in Turbulence, The MIT Press, Cambridge, MA, 1972. 